\title{
MVPNet: Multi-View Point Regression Networks for 3D Object Reconstruction from A Single Image
}

\author{
Jinglu Wang, ${ }^{\dagger}$ Bo Sun, ${ }^{\ddagger *}$ Yan Lu ${ }^{\dagger}$ \\ ${ }^{\dagger}$ Microsoft Research, ${ }^{\ddagger}$ Peking University \\ \{jinglwa, v-bosu, yanlu\}@microsoft.com
}

\begin{abstract}
In this paper, we address the problem of reconstructing an object's surface from a single image using generative networks. First, we represent a 3D surface with an aggregation of dense point clouds from multiple views. Each point cloud is embedded in a regular 2D grid aligned on an image plane of a viewpoint, making the point cloud convolution-favored and ordered so as to fit into deep network architectures. The point clouds can be easily triangulated by exploiting connectivities of the 2D grids to form mesh-based surfaces. Second, we propose an encoder-decoder network that generates such kind of multiple view-dependent point clouds from a single image by regressing their $3 \mathrm{D}$ coordinates and visibilities. We also introduce a novel geometric loss that is able to interpret discrepancy over $3 \mathrm{D}$ surfaces as opposed to $2 \mathrm{D}$ projective planes, resorting to the surface discretization on the constructed meshes. We demonstrate that the multi-view point regression network outperforms state-of-the-art methods with a significant improvement on challenging datasets.
\end{abstract}

\section{Introduction}

3D object reconstruction from a single RGB image is an inherently ill-posed problem as many configurations of shape, texture, lighting, and camera can give rise to the same observed image. Recently, the advanced deep learning models allow for the rethinking of this task as generating realistic samples from underlying distributions. Regular representations are favored by deep convolutional neural networks for dense data sampling, weight sharing, etc. Although meshes are the predominant representations for 3D geometries, their irregular structures are not easy for encoding and decoding. Most extant deep nets (Choy et al. 2016; Tulsiani et al. 2017; Wu et al. 2016; Zhu et al. 2017; Girdhar et al. 2016) employ 3D volumetric grids. However, they suffer from high computational complexity for dense sampling. A few recent methods (Fan, Su, and Guibas 2017; Diamanti, Mitliagkas, and Guibas 2017) advocate the unordered point cloud representation. The unordered property requires additional computation to establish a one-to-one mapping for point pairs. It often yields sparse results because of costly mapping algorithms.

\footnotetext{
${ }^{*}$ The work was done when Bo Sun was an intern at MSR. Copyright (C) 2019, Association for the Advancement of Artificial Intelligence (www.aaai.org). All rights reserved.
}

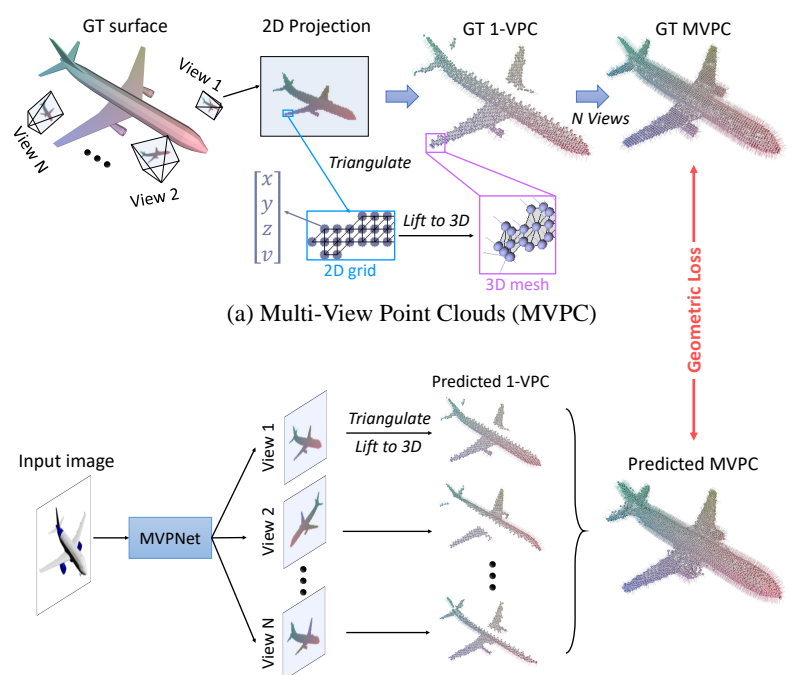

(b) Multi-View Point Network (MVPNet)

Figure 1: (a) A surface is represented by MVPC. Each pixel in a 1-VPC stores the backprojected surface point $(x, y, z)$ from this pixel and its visibility $v$. The stored 3D points are triangulated according to the $2 \mathrm{D}$ grid on the image plane and their normals are shown to indicate surface orientation. (b) Given an RGB image, the MVPNet generates a set of 1 -VPCs and their union forms the predicted MVPC. The geometric loss measures discrepancy between predicted and groundtruth MVPC.

In order to depict dense and detailed surfaces, we introduce an efficient and expressive view-based representation inspired by recent studies on multi-view projections (Kalogerakis et al. 2017; Soltani et al. 2017; Shin, Fowlkes, and Hoiem 2018). In particular, we propose to represent a surface by dense point clouds visible from multiple viewpoints. The arrangement of viewpoints are configured to cover most of the surface. The multi-view point clouds (MVPC) are illustrated in Fig. 1 (a). Each point cloud is stored in a 2D grid embedded in a viewpoint's image plane. A 1-view point cloud (1-VPC) looks like a depth map, but each pixel stores the 3D coordinates and visibility information rather than the depth of the backprojected surface point from this pixel. The backprojection transformation offers a 
one-to-one mapping of point sets in 1-VPCs with equal camera parameters. Meanwhile, local connectivities of the 3D points are introduced from the $2 \mathrm{D}$ grids, which facilitate to form a triangular mesh based on such backprojected points.

Accordingly, the surface reconstruction problem is formulated as the regression of values stored in MVPC. We employ an encoder-decoder network as a conditional sampler to generate the underlying MVPC, as shown in Fig. 1 (b). The encoder extracts image features and combines them with different viewpoints' features respectively. The decoder consists of multiple weight-shared branches, each of which generates a view-dependent point cloud. The union of all 1 -VPCs forms the final MVPC. We propose a novel geometric loss that measures discrepancies over real 3D surfaces as opposed to 2D planes. Unlike previous view-based methods processing features in 2D projective spaces (i.e., image planes) and neglecting the information loss through dimension reduction from $3 \mathrm{D}$ to $2 \mathrm{D}$, the proposed MVPC allow us to discretize integrals of surface variations over the constructed triangular mesh. The geometric loss integrating volume variations, prediction confidences and multi-view consistencies contributes to high reconstruction performance.

\section{Related Work}

Mesh-based methods. Mesh representation has been extensively used to improve and manipulate surface interfaces. In particular, surface reconstruction is usually posed to deform an initial mesh to minimize a variational energy functional in the spirit of data fidelity. (Delaunoy and Prados 2011; Pons, Keriven, and Faugeras 2007; Wang et al. ; Liu et al. 2015) are the pioneers of reconstructing meshbased surface from multi-views. These deformable mesh methods calculate the integral over the whole surface and thus capture complete properties on the surface. However, irregular connectivities of mesh representation make it difficult to leverage the advance of convolutional architectures. Recent methods (Pontes et al. 2017) use linear combinations of a dictionary of CAD models and learn the parameters of the combination to represent the models, which are limited to the capacity of the constructed dictionary. We are inspired by variational methods that have geometric interpretations for optimization formulation. Important geometric clues are integrated into the loss function, which contributes a superior performance significantly.

Voxel-based methods. When learning methods dominate the recognition tasks, volumetric representation (Girdhar et al. 2016; Wu et al. 2016; Choy et al. 2016; Wu et al. 2017; Tulsiani et al. 2017) is more favored because of its regular grid-like structure that suites convolutional operations. Tulsiani and Zhou (Tulsiani et al. 2017) formulate a differentiable ray consistency term to enforce view consistency on the voxels with the supervision of multi-view observation. 3D-R2N2 (Choy et al. 2016) learns to aggregate voxel occupancy from sequential input images and can obtain robust results. Voxel-based methods are limited by the cubic growth rate of both memory and computation time, leading to low-resolution of grids.
View-based methods. As the drawbacks of voxel-based CNNs are obvious, some methods adopt view-based representations. They project surfaces on image planes with regular 2D grids that allows planar convolution. A few methods (Park et al. 2017; Zhou et al. 2016) achieve impressive results in synthesizing novel views from a single view. Tatarchenko et al (Tatarchenko, Dosovitskiy, and Brox 2016) utilize CNNs to infer images and depth maps of arbitrary views given an RGB image, and then fuse the depth maps to yield a 3D surface. Soltani et al (Soltani et al. 2017) synthesize multi-view depth maps from a single or multiple depth maps. Since depth maps inherently contain geometric information, our task, which takes a single RGB image as an input is much more challenging. Lin et al (Lin, Kong, and Lucey 2018) also generate points of multiple views with a generative network. These methods all focus on predicting the intermediate information in 2D projective planar spaces yet ignore real 3D spatial correlation and multi-view consistency. Our method incorporates the spatial correlation of surface points and further enforces multi-view consistency to achieve more accurate and robust reconstructions.

Point-based methods. Some methods generate an unordered point cloud from an image by deep learning. Su et al (Fan, Su, and Guibas 2017) are the first to study the problem. The unordered property of a point cloud enjoys high flexibility (Qi et al. 2017), but it increases computational complexity due to lack of correspondences. This makes such methods not scalable, resulting in sparse points.

\section{Approach}

In this section, we first formally introduce the MVPC representation for depicting 3D surfaces efficiently and expressively. Then, we detail the MVPNet architecture and the geometric loss for generating the underlying MVPC conditioned on an input image.

\section{MVPC Representation}

An object's surface $\mathcal{S}$ is considered as an aggregation of partial surfaces $\bigcup_{i=1}^{N} S_{i}$ visible from a set of predefined viewpoints $\left\{\mathbf{c}_{i} \mid i=1, \ldots, N\right\}$. Each partial surfaces $S_{i}$ is discretized and parameterized by the aligned $2 \mathrm{D}$ grid on the image plane of $\mathbf{c}_{i}$, as shown in Fig. 1 (a). Each pixel $x_{k}$ on the grid stores the $3 \mathrm{D}$ point $\mathbf{x}_{k}=\left(x_{k}, y_{k}, z_{k}\right)$ backprojected from $x_{k}$ onto $\mathcal{S}$ and the visibility $v_{k}^{i}$ of $\mathbf{x}_{k}$ from $\mathbf{c}_{i} . v_{k}^{i}$ is set to 1 if $\mathbf{x}_{k}$ is visible from $\mathbf{c}_{i}$, otherwise 0 . The visible $3 \mathrm{D}$ points are triangulated by connecting them with the $2 \mathrm{D}$ grid's horizontal, vertical, and one of the diagonal edges to form a mesh-based surface. Such multiple view-dependent parameterized surfaces are named multi-view point clouds, MVPC in short, $\mathcal{M}=\bigcup_{i=0}^{N} M_{i}$, where $M_{i}$ denotes a 1-view point cloud, 1-VPC in short. Let $\mathcal{X}=\left\{\mathbf{x}_{k}\right\}$ denote all the 3D points in $\mathcal{M}$.

MVPC inherit the advantage of efficiency from general view-based representations. Unlike volumetric representation using costly 3D convolution, 2D convolution is performed on the 2D grids, which encourages higher resolutions for denser surface sampling. Meanwhile, MVPC 


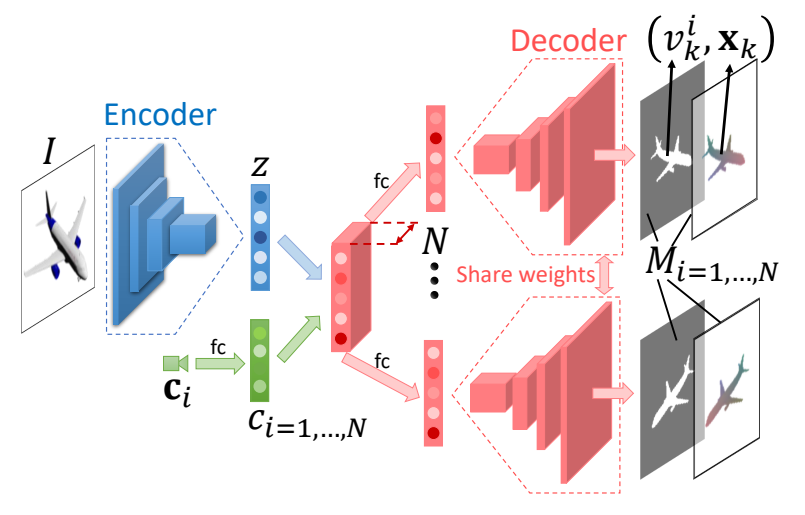

Figure 2: MVPNet architecture. Given an input image $I$, MVPNet consisting of an encoder and a decoder regresses the $N$ 1-VPCs $\left\{M_{i}\right\}$ for $\left\{\mathbf{c}_{i}\right\}, i=1, \ldots, N$ respectively. $N$ concatenated features $\left(z, c_{i}\right)$ are fed into $N$ branches of the decoder, of which the branches share weights.

encode one-to-one mapping of predicted points $\mathcal{X}$ and groudtruth points $\widetilde{\mathcal{X}}$ explicitly. Induced by the same viewpoint $\mathbf{c}_{i}$, pixels with the same $2 \mathrm{D}$ coordinates $x_{k}$ are defined to store the same surface point $\mathbf{x}_{k}$. In other words, the groundtruth and predicted 1-VPC of the same viewpoint store the points in the same order. Compared to unordered point cloud representations that require additional computation to construct point-wise mapping, MVPC have superior performance in computation.

MVPC express not a simple combination of multi-view projections but a discrete approximation to a real 3D surface. On the one hand, A triangular mesh is constructed for each $1-\mathrm{VPC}$, and thus we can formulate losses based on geometries on $3 \mathrm{D}$ surfaces rather than on $2 \mathrm{D}$ projections. Note that the edges inherited from 2D grids are not all real in 3D, e.g., edges connecting points on depth discontinuities are fake. We deal with the fake edges by penalizing them largely in the loss formulation. On the other hand, we carefully select relatively few yet evenly distributed viewpoints on a viewing sphere that can cover most of the targeting surface. Different numbers of viewpoints are discussed in the experiment section. We also consider multi-view consistency constraints in overlap regions to improve the expressiveness of MVPC.

\section{MVPNet Architecture}

We exploit an encoder-decoder generative network architecture and incorporate camera parameters into the network to generate view-dependent point clouds. The network architecture is illustrated in Fig. 2. The encoder learns to map an image $I$ to an embedding space to obtain a latent feature $z$. Each camera matrix $\mathbf{c}_{i}$ is first transformed to a higherdimensional hidden representation $c_{i}$, serving as a view indicator, and then is concatenated with $z$ to get $\left(z, c_{i}\right)$. The decoder that converts $\left(z, c_{i}\right)$ to a 1-VPC $M_{i}$ indicated $\mathbf{c}_{i}$ learns the projective transformation and space completion. The decoder shares weights among $N$ branches. The output
MVPC $\mathcal{M}=\bigcup_{i=1}^{N} M_{i}$ is of shape $N \times H \times W \times 4$, where $H$ and $W$ denote the height and width of a 1-VPC. The last channel corresponds to a $3 \mathrm{D}$ coordinate $\mathbf{x}_{k}=\left(x_{k}, y_{k}, z_{k}\right)$ and visibility $v_{k}^{i}$ of a point $\mathbf{x}_{k}$.

The encoder is a composition of convolution and leaky ReLU layers. The camera parameters are encoded with fully connected layers. The decoder contains a sequence of transposed-convolution and leaky ReLU layers. The last layer is activated with the tanh functions, responsible for regressing $3 \mathrm{D}$ coordinates and visibilities of points. Implementation details are described in the experiment section.

\section{Geometric Loss}

While most point generation methods (Fan, Su, and Guibas 2017; Lin, Kong, and Lucey 2018; Soltani et al. 2017) adopt point-wise distance metrics, they disregard geometric characteristics of surfaces. These networks attempt to predict "mean" shapes (Fan, Su, and Guibas 2017), failing to preserve fine details.

We propose a geometric loss (GeoLoss) that is able to capture variances over 3D surfaces rather than over sparse point sets or 2D projective planes. We expand the GeoLoss to be differentiable for neuron networks and also to be robust against noise and incompletion. The GeoLoss is made up of three components:

$$
\mathcal{L}_{G e o}=\mathcal{L}_{p t d}+\alpha \mathcal{L}_{v o l}+\beta \mathcal{L}_{m v}
$$

where $\mathcal{L}_{p t d}$ is the sum of distances between corresponding point pairs, $\mathcal{L}_{\text {vol }}$ denotes the quasi-volume term measuring discrepancy of local volumes, and $\mathcal{L}_{m v}$ is the multi-view consistency term. Coefficients $\alpha$ and $\beta$ are the weights balancing different losses.

Point-wise distance term. The points in groundtruth and predicted 1-VPC have a one-to-one mapping according to the definition of MVPC, illustrated in Fig. 3 (a). 2D pixels with equal 2D coordinates are defined to store the same surface point induced by the same viewpoint. Therefore, the sum of point-wise distances for groundtruth and predicted $1-\mathrm{VPC}$ is the L2 loss. The total sum of point-wise distances of MVPC is given by:

$$
\mathcal{L}_{p t d}=\sum_{i}^{N} \sum_{x \in M_{i}}\left\|M_{i}(x)-\widetilde{M}_{i}(x)\right\|_{2}
$$

where $x$ is a $2 \mathrm{D}$ pixel, $M_{i}(x)$ and $\widetilde{M}_{i}(x)$ denotes the 3D coordinates stored in predicted 1-VPC $M_{i}$ and groundtruth 1 -VPC $\widetilde{M}_{i}$ at $x$. Here we take the visibility into account by setting $\widetilde{M}_{i}(x)$ to an infinite point $\mathbb{F}^{3}$ (a point at the far clipping plane practically).

Neural networks tend to predict a mean shape averaging out the space of uncertainty using L2 or L1 loss. Point-wise distances neglecting local interactions do not fully express geometric discrepancy between surfaces. Moreover, this metric may give rise to erroneous reconstructions around occluding contours, because minor errors on $2 \mathrm{D}$ projective planes lead to large 3D deviations at depth continuities. 


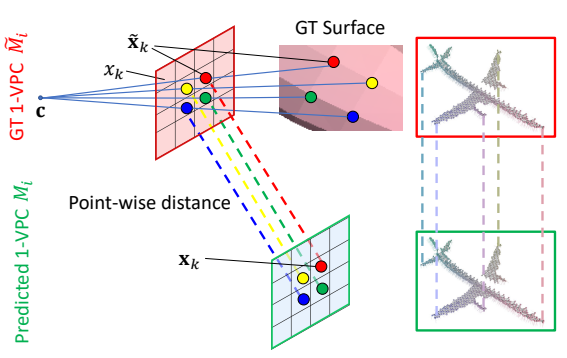

(a) Point-wise distance term.

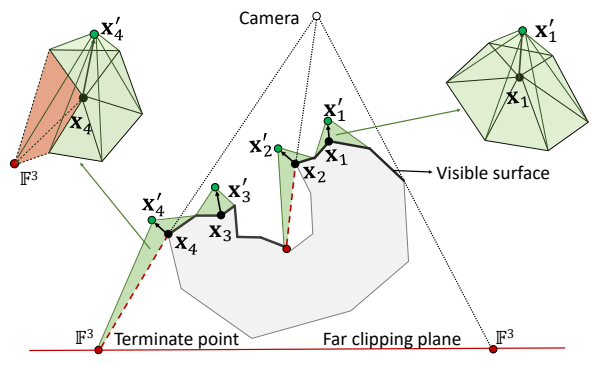

(b) Quasi-volume term.

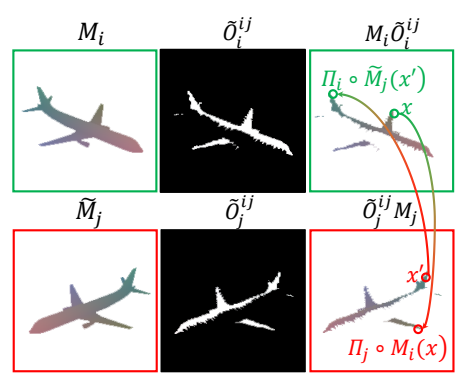

(c) Multi-view consistency term.

Figure 3: Loss functions. (a) Point-wise distances for 1-VPC. Induced by the same viewpoint, groundtruth and predicted points $\left(\widetilde{\mathbf{x}}_{k}\right.$ and $\left.\mathbf{x}_{k}\right)$ stored in $x_{k}$ indicate the same surface point. (b) Quasi-volume discrepancy for typical examples. The local volume discrepancies of points around depth discontinuities, e.g., $\mathbf{x}_{2}$ and $\mathbf{x}_{4}$, are largely penalized by keeping fake connectivities (red dashed lines). (c) $\widetilde{O}_{i}^{i j}$ is the projection on view $i$ of the overlap region visible from view $i$ and $j$. $\widetilde{O}_{j}^{i j}$ is the projected overlap region on view $j$. Considering the overlap region, the multi-view consistency term minimizes the sum of distances between 3D points stored in pixels $x \in M_{i}$ and its reprojected pixel $\Pi_{j} \circ M_{i}(x) \in \widetilde{M}_{j}$, and verse visa.

Quasi-volume term. The limitation of point-wise distance metrics motivates us to formulate discrepancies over surfaces. Inspired by the volume-preserving constraints used in variational surface deformation (Eckstein et al. 2007), we propose a quasi-volume discrepancy metric to better describe the surface discrepancy. This term is able to characterize fine details and deal with occluding contours.

Let us first define the volume discrepancy between predicted and groundtruth continuous surfaces:

$$
\mathcal{L}_{\text {vol }}(\mathcal{S}, \widetilde{\mathcal{S}})=\int_{\mathcal{S}}(\mathbf{x}-\widetilde{\mathbf{x}}) \cdot \mathbf{n} \mathrm{d} \mathbf{x}
$$

where $\mathbf{x}$ and $\widetilde{\mathbf{x}}$ are $3 \mathrm{D}$ points on predicted surface $\mathcal{S}$ and groundtruth surface $\widetilde{\mathcal{S}}, \mathrm{d} \mathbf{x}$ is an area element of a surface and $\mathbf{n}$ is the outward normal to the surface at point $\mathbf{x},(\cdot)$ denotes the inner product operator.

The discrete volume discrepancy of the MVPC representation can be deduced as:

$$
\mathcal{L}_{\text {vol }}=\sum_{i}^{N} \sum_{x \in M_{i}} \widetilde{V}_{i}(x)\left(M_{i}(x)-\widetilde{M}_{i}(x)\right) \cdot \widetilde{\mathbf{N}}_{i}(x)
$$

where $\widetilde{V}_{i}$ is the groundtruth visibility map and $\widetilde{\mathbf{N}}_{i}$ is the groundtruth area-weighted normal map of view $i$. Formally, for each pixel $x$ with its backprojected surface point $\mathbf{x}$, the normal map of view $i$ is $\mathbf{N}_{i}(x)=\sum_{\Delta \in \Omega(\mathbf{x})}|\Delta| \mathbf{n}(\mathbf{x})$, where $\Delta$ denotes a mesh triangle, $\Omega(\mathbf{x})$ contains the 1-ring triangles around point $\mathbf{x}, \mathbf{n}(\mathbf{x})$ is the outward normal at point $\mathbf{x}$. A detailed proof is presented in the supplemental material. Note that we use the groundtruth visibility $\widetilde{V}_{i}(\cdot)$, and thus add a cross entropy loss accounting for the visibilities.

Equation 4 formulates the volume discrepancy for visible parts. We complement it with invisible parts, and name it as quasi-volume discrepancy, as illustrated in Fig. 3 (b). We assign the background pixels with a terminate point $\mathbb{F}^{3}$ at far clipping plane. Thus, the points at boundaries of $M_{i}$ will achieve a large discrepancy gain, which means they are of high weights in the loss function, e.g., $\mathbf{x}_{4}$ in Fig. 3 (b). Similarly, points at occluding contours experience large volume loss, e.g., $\mathbf{x}_{2}$ in Fig. 3 (b). The quasi-volume term implicitly handles the challenges introduced by occluding contours.

Multi-view consistency term. Partial surfaces of an object visible from different viewpoints may have overlap, which can be reached by letting points from different views attract one another. The consistency serves as links between groundtruth and predicted 3D points stored in a pair of corresponding pixels from two different views. Fig. 3 (c) shows an example for two views. Note that the consistency only exists at overlap regions. We first compute the projected overlap region $\widetilde{O}_{i}^{i j}$ on view $i$ by rendering groundtruth 1-VPC $\widetilde{M}_{j}$ on view $i$, and get $\widetilde{O}_{j}^{i j}$ by rendering $\widetilde{M}_{i}$ on view $j$. We minimize the sum of two distances between the stored 3D points in two corresponding pixels and their reprojected pixels in the other view. For each pixel $x$ in $\widetilde{O}_{i}^{i j}$, the predicted 3D coordinate is $M_{i}(x)$. The reprojected pixel on view $j$ is $\Pi_{j} \circ M_{i}(x)$, where $\Pi_{j}$ denotes the projection matrix of view $j$. Similarly, the pixel $x^{\prime}$ at groundtruth 1 -VPC $\widetilde{M}_{j}$ corresponds to the pixel $\Pi_{i} \circ \widetilde{M}_{j}\left(x^{\prime}\right)$ in the predicted 1-VPC $M_{i}$. Therefore, the multi-view consistency term takes the form:

$$
\begin{aligned}
\mathcal{L}_{m v}= & \sum_{i, j}\left(\sum_{x \in \widetilde{O}_{i}^{i j}}\left\|M_{i}(x)-\widetilde{M}_{j}\left(\Pi_{j} \circ M_{i}(x)\right)\right\|_{2}\right. \\
& \left.+\sum_{x \in \widetilde{O}_{j}^{i j}}\left\|\widetilde{M}_{j}(x)-M_{i}\left(\Pi_{i} \circ \widetilde{M}_{j}(x)\right)\right\|_{2}\right)
\end{aligned}
$$

The multi-view consistency term does not directly minimize distances between two predicted 1-VPCs but leverages the correspondences between predictions and groundtruths. This is because erroneous 3D coordinates in predictions will introduce false correspondences, resulting in divergence or falling into a trivial solution.

\section{Experiment}

\section{Implementation}

We show the architecture of MVPNet in Fig. 2. The input RGB image is of size $128 \times 128$. The output surface coor- 
Table 1: Quantitative comparison to the state-of-the-arts with per-category voxel IoU.

\begin{tabular}{|c|c|c|c|c|c|c|c|c|c|c|c|c|c|c|c|}
\hline & & plane & bench & cabinet & car & chair & display & lamp & speaker & firearm & couch & table & phone & vessel & mean \\
\hline \multirow{4}{*}{$\begin{array}{l}\overline{\mathbb{v}} \\
\stackrel{ٍ}{\rho}\end{array}$} & R2N2(Choy et al. 2016)(1 view) & 0.513 & 0.421 & 0.716 & 0.798 & 0.466 & 0.468 & 0.381 & 0.662 & 0.544 & 0.628 & 0.513 & 0.661 & 0.513 & 0.56 \\
\hline & R2N2(Choy et al. 2016)(5 views) & 0.561 & 0.527 & 0.772 & 0.836 & 0.550 & 0.565 & 0.421 & 0.717 & 0.600 & 0.706 & 0.580 & 0.754 & 0.610 & 0.630 \\
\hline & PTN-Comb(Yan et al. 2016) & 0.584 & 0.508 & 0.711 & 0.738 & 0.470 & 0.547 & 0.422 & 0.587 & 0.610 & 0.653 & 0.515 & 0.773 & 0.551 & 0.590 \\
\hline & CNN-Vol(Yan et al. 2016) & 0.575 & 0.514 & 0.697 & 0.735 & 0.445 & 0.539 & 0.386 & 0.548 & 0.603 & 0.647 & 0.514 & 0.769 & 0.5445 & 0.578 \\
\hline \multirow{2}{*}{$\begin{array}{l}\vec{\Xi} \\
\vdots \\
\vdots\end{array}$} & Soltani(Soltani et al. 2017) & 0.587 & 0.524 & 0.698 & 0.743 & 0.529 & 0.679 & 0.480 & 0.586 & 0.635 & 0.59 & 0.593 & 0.789 & 0.604 & 0.618 \\
\hline & $\mathrm{Su}(\mathrm{Fan}, \mathrm{Su}$, and Guibas 2017) & 0.601 & 0.55 & 0.771 & 0.831 & 0.544 & 0.552 & 0.462 & 0.737 & 0.604 & 0.708 & 0.606 & 0.749 & 0.611 & 0.640 \\
\hline \multirow{3}{*}{ 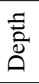 } & $\operatorname{GeoLoss}(N=4)$ & 0.655 & 0.578 & 0.664 & 0.709 & 0.546 & 0.653 & 0.486 & 0.573 & 0.676 & 0.630 & 0.561 & 0.783 & 0.633 & 0.627 \\
\hline & $\operatorname{GeoLoss}(N=6)$ & 0.624 & 0.579 & 0.677 & 0.719 & 0.543 & 0.636 & 0.498 & 0.578 & 0.682 & 0.636 & 0.548 & 0.800 & 0.643 & 0.628 \\
\hline & $\operatorname{GeoLoss}(N=8)$ & 0.622 & 0.576 & 0.691 & 0.724 & 0.540 & 0.643 & 0.501 & 0.590 & 0.684 & 0.647 & 0.534 & 0.788 & 0.640 & 0.629 \\
\hline \multirow{4}{*}{ 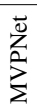 } & $\operatorname{PtLoss}(N=6)$ & 0.474 & 0.459 & 0.573 & 0.704 & 0.436 & 0.558 & 0.375 & 0.496 & 0.519 & 0.567 & 0.432 & 0.691 & 0.558 & 0.526 \\
\hline & $\operatorname{GeoLoss}(N=4)$ & 0.666 & 0.622 & 0.693 & 0.786 & 0.616 & 0.653 & 0.510 & 0.599 & 0.696 & 0.690 & 0.635 & 0.811 & 0.663 & 0.665 \\
\hline & $\operatorname{GeoLoss}(N=6)$ & 0.678 & 0.623 & 0.685 & 0.788 & 0.627 & 0.681 & 0.523 & 0.602 & 0.693 & 0.701 & 0.652 & 0.814 & 0.659 & 0.671 \\
\hline & $\operatorname{GeoLoss}(N=8)$ & 0.667 & 0.610 & 0.686 & 0.782 & 0.609 & 0.667 & 0.507 & 0.596 & 0.688 & 0.686 & 0.641 & 0.809 & 0.661 & 0.662 \\
\hline
\end{tabular}

Table 2: Quantitative comparison to point-based methods using the chamfer distance metric. All numbers are scaled by 0.01 .

\begin{tabular}{|c|c|c|c|c|c|c|c|c|c|c|c|c|c|c|}
\hline & plane & bench & cabinet & car & chair & display & lamp & speaker & firearm & couch & table & phone & vessel & mean \\
\hline Su(Fan, Su, and Guibas 2017) & 1.395 & 1.899 & 2.454 & 1.927 & 2.121 & 2.127 & 2.280 & 3.000 & 1.337 & 2.688 & 2.052 & 1.753 & 2.064 & 2.084 \\
\hline Lin(Lin, Kong, and Lucey 2018) & 1.418 & 1.622 & 1.443 & 1.254 & 1.964 & 1.640 & 3.547 & 2.039 & 1.400 & 1.670 & 1.655 & 1.569 & 1.682 & 1.761 \\
\hline Soltani(Soltani et al. 2017) & 0.167 & 0.165 & 0.122 & 0.026 & 0.277 & 0.085 & 1.814 & 0.163 & 0.107 & 0.138 & 0.226 & 0.258 & 0.102 & 0.28 \\
\hline $\operatorname{MVPNet}(N=4)$ & 0.045 & 0.084 & 0.063 & 0.042 & 0.086 & 0.065 & 0.561 & 0.163 & 0.104 & 0.082 & 0.070 & 0.046 & 0.060 & 0.113 \\
\hline $\operatorname{MVPNet}(N=6)$ & 0.041 & 0.079 & 0.060 & 0.041 & 0.085 & 0.053 & 0.421 & 0.152 & 0.093 & 0.070 & 0.069 & 0.038 & 0.050 & 0.096 \\
\hline $\operatorname{MVPNet}(N=8)$ & 0.044 & 0.085 & 0.058 & 0.040 & 0.103 & 0.050 & 0.494 & 0.153 & 0.113 & 0.083 & 0.075 & 0.039 & 0.059 & 0.107 \\
\hline
\end{tabular}

dinate maps is of shape $N \times 128 \times 128 \times 4$. The encoder consists of five convolution (conv) layers with numbers of channels $\{32,64,128,256,512\}$, kernel sizes $\{3,3,3,3$, $3\}$, and strides $\{2,2,2,2,2\}$, and two fully connected (fc) layers with numbers of neurons $\{4096,2048\}$. The camera matrix $\mathbf{c}$ is encoded with two fc layers with numbers of neurons $\{64,512\}$. The decoder part takes the concatenated feature $\left(z, c_{i}\right)$ as input and generates a surface coordinate map for each viewpoint. The structure of the decoder is mirrored to the encoder, consisting of two fc layers and five transposed-convolution (also known as "deconv") layers for up-sampling. We add the last conv layer with the number of channels 4 and kernel size 1 to generate 4-channel output. Batch normalization (Ioffe and Szegedy 2015) is not performed because we observe the training process is smooth. Leaky ReLU activation with a negative slope of 0.2 is applied after all conv layers except the last one which is followed by the tanh layer.

We train the network with Tensorflow (Abadi et al. 2016) on a Nvidia TitanX GPU with a minibatch of 32. We use Adam optimizer (Kingma and $\mathrm{Ba}$ 2014) with a learning rate of 0.0001 . The training procedure takes 100,000 iterations. The coefficients $\alpha$ and $\beta$ of GeoLoss is set to 100 and 1 respectively after 10000 iterations and both to 0 before, because the initial point clouds are noisy and the computed volume discrepancy and consistency term are not reliable.

\section{Dataset}

We leverage the ShapeNet (Chang et al. 2015) dataset, which contains a large volume of clean CAD models for our experiments. We setup two datasets for single-class and multiclass cases. The chair category (ShapeNet-Chair) is used for single-class processing since it is ubiquitously evaluated in previous methods. For the multi-class dataset, we use 13 major classes as the 3D-R2N2 (Choy et al. 2016) set, listed in Table 1, named as ShapeNet-13. The datasets are split into training and testing sets with the fraction 0.8/0.2.

To obtain input RGB images, we render each 3D model for 24 viewpoints which are randomly sampled with an elevation ranging from $(-20,20)$, an azimuth ranging from $(0$, $360)$ degrees, and a radius ranging from $(0.6,2.3)$. Note that all models are normalized by their bounding spheres' radius.

Viewpoint arrangement. For the viewpoint arrangement of the output MVPC, we approximately maximize the coverage of the "mean" shape (the unit sphere) of all objects with respect to $N$. The $N(4,6,8)$ viewpoints are located at vertices of a tetrahedron, octahedron, and cube, respectively. All the viewpoints look at the origin. Orthogonal projection is used to avoid additional perspective distortion. We calculate the average surface coverage by counting the number of visible points in groundtruth models, which are $97.2 \%$, $97.7 \%$ and $98.0 \%$ for $N=4,6,8$ respectively. The performances of different viewpoint settings are reported.

\section{Reconstruction Result}

Both qualitative and quantitative results of the reconstruction are presented. We compare our method to two collections of state-of-the-art methods according to the final result representations, namely, point clouds and volumetric grids.

Comparison to point generation methods. We compare our method to the state-of-the-art point generation methods using both an unordered point cloud representation (Fan, Su, and Guibas 2017) and view-based representations (Soltani et al. 2017; Lin, Kong, and Lucey 2018) on the ShapeNet-13 dataset. Note that the methods proposed by Su et al (Fan, $\mathrm{Su}$, and Guibas 2017), Lin et al (Lin, Kong, and Lucey 2018) and us take a single RGB image as the input, while Soltani et al (Soltani et al. 2017) use depth maps as input which may contain more geometric information. We use Intersection-of-Union (IoU) of voxel occupancy for evaluating the reconstruction accuracy as most methods do. The 


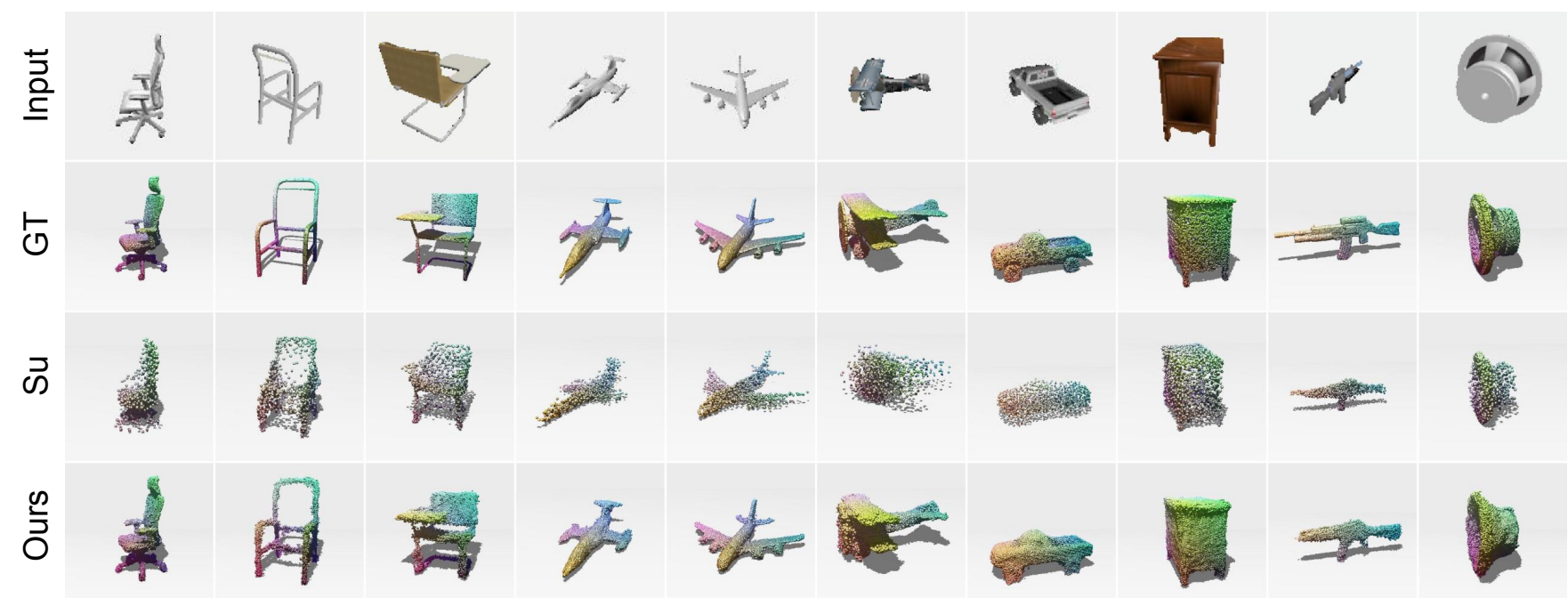

Figure 4: Qualitative comparison to point generation method. Compared with Su et al (Fan, Su, and Guibas 2017), our method preserves more fine details and recovers better concave structures.

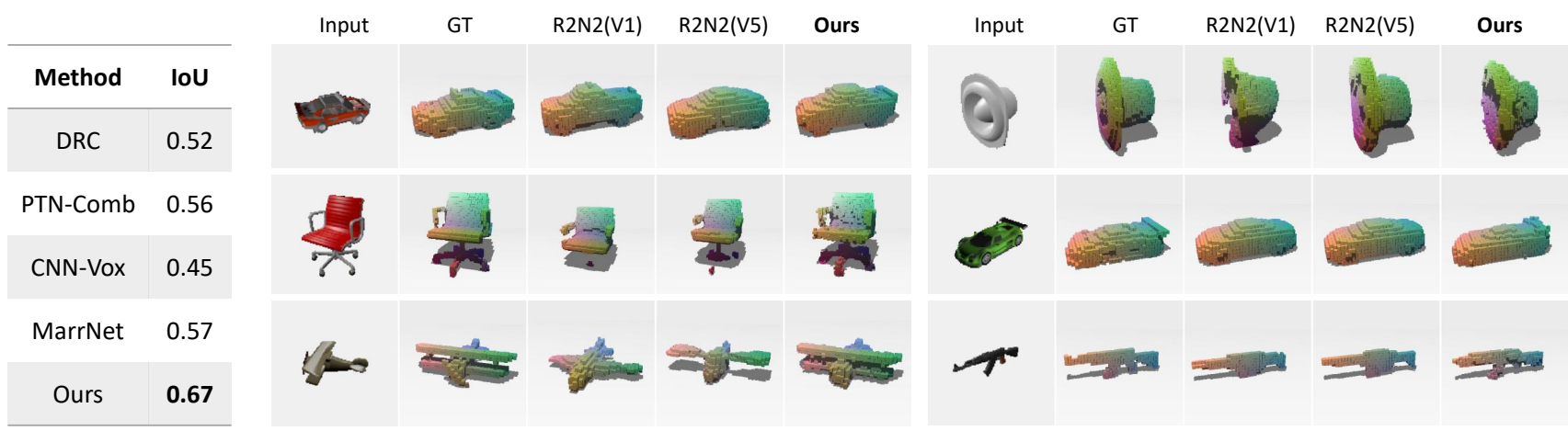

(a) IoU on ShapeNet-Chair

(b) Visual compare to 3D-R2N2 on ShapeNet-13

Figure 5: Comparison to voxel-based methods. (a) Quantitative comparison on ShapeNet-Chair. (b) Qualitative comparison to 3D-R2N2 (Choy et al. 2016) on ShapeNet-13. Our method with a single image generates more detailed results than 3D-R2N2 with 5 input images (V5).

per-class IoU statistics are reported in Table 1. Our results of different numbers of viewpoints $(N=4,6,8)$ are reported for discussion. For a fair comparison, we adopt the model proposed in (Soltani et al. 2017) without class supervision and post-processing. All the results are generated from the trained model provided by the authors (Soltani et al. 2017; Fan, Su, and Guibas 2017).

Our results using GeoLoss outperform the previous methods on 9 out of 13 classes with 6 and 8 viewpoints. The results of 6 viewpoints achieve best on 7 classes, which demonstrates that 6 viewpoints are sufficient to cover most objects and suppress error propagation in multi-view learning. Note that our network with the GeoLoss achieves significantly better results on classes with a lot of thin and complex structures, such as planes $(+17 \%)$, chairs $(+7 \%)$, and lamps $(+6 \%)$. This is because our method exacts at capturing fine details by minimizing the GeoLoss which interprets the variance over 3D surfaces rather than sparse points or 2D projective planes. The importance of GeoLoss is demonstrated by comparing with the results using only point-wise distance term (PtLoss). We find that the results from $\mathrm{Ge}$ oLoss are about $10 \%$ higher than the ones from PtLoss with 6 viewpoints (best in GeoLoss). We use GeoLoss in the following experiments. Our reconstruction accuracy for classes with simple structures, e.g., cabinet, car and display, are slightly lower than $\mathrm{Su}$ (Fan, Su, and Guibas 2017) and Soltani (Soltani et al. 2017), because their net architectures are more complex ("hourglass" structure in Su (Fan, Su, and Guibas 2017) and "ResNet blocks" in Soltani (Soltani et al. 2017)) and predict better "mean" shapes.

To evaluate the faithfulness of the generated point to the groundtruth surface, we compute the Chamfer Distance (CD) (Fan, Su, and Guibas 2017) between the prediction and the densely sampled points from groundtruth meshes. CD is 
a common measure of the distance between two point sets, which is defined by summing up the distances between each source point to its nearest point in the target point set. The groundtruth points of size 100,000 are uniformly sampled on the surface. The CD evaluation is reported in Table 2. Our method is superior to the previous methods on most classes (12/13) by a large margin. Same as in IoU evaluation, 6 viewpoints get the best on 10 classes. The multiview point clouds generated by our network possess high density and the geometric loss enforces local spatial coherence. The unordered point generation method (Fan, $\mathrm{Su}$, and Guibas 2017) gets sparse point clouds which are limited to characterizing enough details, leading to large chamfer distances. The method proposed by Soltani et al (Soltani et al. 2017) also obtains small distances since it generates points with many more (20) depth maps.

For qualitative comparison, we present several typical examples in Fig. 4. Our method is able to produce much denser points $(\sim 15 \mathrm{k})$, while the method proposed by $\mathrm{Su}$ (Fan, $\mathrm{Su}$, and Guibas 2017) limits the point cloud size to 1024. Our method is superior in recovering fine details (see chair backs, plane tails and car wheels) and dealing with concave structures, such as car trunk and two layers of plane wings. The geometric loss that handles occlusion encourages the improvement on concave shapes. More results of ours are shown in supplemental materials.

Comparison to voxel-based methods. We compare the proposed method to the state-of-the-art voxel-based methods, i.e., 3D-R2N2 (Choy et al. 2016), DRC (Tulsiani et al. 2017), two models of PTN (Yan et al. 2016) (PTN-Comb, CNN-Vox), and MarreNet (Wu et al. 2017). These methods directly use 3D volumetric representation and usually compute the IoU for evaluation. Since our results form dense point clouds, we convert them to $(32 \times 32 \times 32)$ grids as $\mathrm{Su}$ et al (Fan, Su, and Guibas 2017) do. For single class model, our method achieves much higher IoU (0.667) than the highest IoU (0.57) among the state-of-the-art methods on the ShapeNet-Chair dataset, shown in Fig. 5 (a). For the multi-class results, we report per-category IoU in Table 1 on ShapeNet-13 dataset. The qualitative comparison to 3DR2N2 is shown in Fig. 5 (b). We show that our method preserves more fine details, such as legs of chairs, wings of planes, and holders of firearms.

Comparison to depth regression. Here we show our findings that directly regressing 3D coordinates has advantages over regressing depths. To compare 3D coordinates and depth regression, we adopt the same network architecture but the last layer and use the same GeoLoss. The channel numbers of the last layers are 3 and 1 for regressing coordinates and depths respectively. As reported in Table 1, the depth regression generates reasonable results, but the accuracy is about $4 \%$ lower than coordinate regression. This is because searching a gradient decent move in 3D space with an arbitrary direction is more flexible and stable than searching in one fixed direction considering the loss of the 3D space (rather than 1D depth loss), especially on occluding contours. With the same 3D volume loss descent, the 3D point needs a small move in the steepest direction, while the

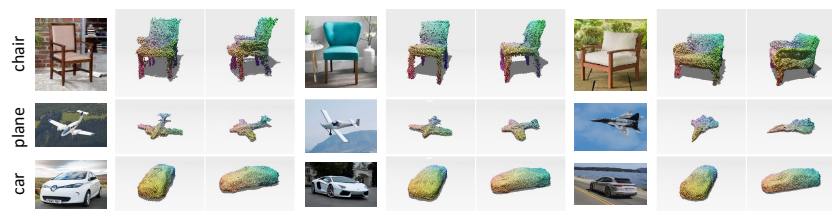

Figure 6: Reconstruction results on real word data.

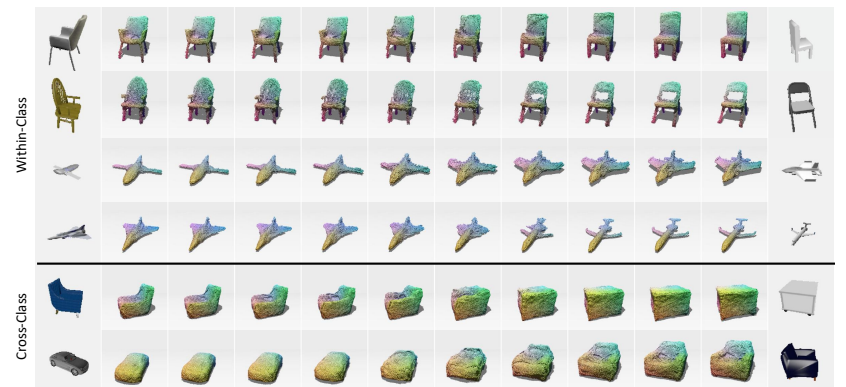

Figure 7: Reconstructions for linear interpolation of two learned latent features within and across classes respectively.

depth needs an extremely large move in the fixed direction. Thus, 3D coordinates are easier to learn than depths. In addition, the complexity does not grow much because only the last layer of the decoder is different.

Results on real dataset. We show our model works well on natural images without additional input. To adjust our model to real-world images, we synthesize the training data by augmenting the input images with random crops from the PASCAL VOC 2012 dataset (Everingham et al. 2011) as (Tatarchenko, Dosovitskiy, and Brox 2016) do. We show that the proposed method yields reasonable results in Fig. 6.

Application. We show the generative representation of the learned features using linear interpolation in Fig. 7. We can see clear and gradual transitions of the generated point clouds, indicating the learned feature space to be sufficiently representative and smooth. More results of discriminative representations are presented in the supplemental material.

\section{Conclusions}

We have presented the MVPNet for regressing dense 3D point clouds of an object from a single image. The point regression achieves state-of-the-art performance resorting to the MVPC representation and the geometric loss. The MVPC express an object's surface with view-dependent point clouds that are embedded in regular 2D grids, which easily fit into CNN-based architectures. Also, the one-toone mapping from $2 \mathrm{D}$ pixels to reprojected $3 \mathrm{D}$ points makes these points in 1-VPC ordered, which accelerate the loss computation. Although the dimension of the data embedding space is reduced from $3 \mathrm{D}$ space to $2 \mathrm{D}$ projective planes, we propose the geometric loss that integrates variances over the $3 \mathrm{D}$ surfaces instead of the $2 \mathrm{D}$ projective planes. The experiments demonstrate the geometric loss significantly improves the reconstruction accuracy. 


\section{References}

Abadi, M.; Agarwal, A.; Barham, P.; Brevdo, E.; Chen, Z.; Citro, C.; Corrado, G. S.; Davis, A.; Dean, J.; Devin, M.; et al. 2016. Tensorflow: Large-scale machine learning on heterogeneous distributed systems. arXiv preprint arXiv:1603.04467.

Chang, A. X.; Funkhouser, T.; Guibas, L.; Hanrahan, P.; Huang, Q.; Li, Z.; Savarese, S.; Savva, M.; Song, S.; Su, H.; et al. 2015. Shapenet: An information-rich 3d model repository. arXiv preprint arXiv:1512.03012.

Choy, C. B.; Xu, D.; Gwak, J.; Chen, K.; and Savarese, S. 2016. 3d-r2n2: A unified approach for single and multi-view $3 \mathrm{~d}$ object reconstruction. In $E C C V$.

Delaunoy, A., and Prados, E. 2011. Gradient flows for optimizing triangular mesh-based surfaces: Applications to $3 \mathrm{~d}$ reconstruction problems dealing with visibility. International journal of computer vision (IJCV) 95(2):100-123.

Diamanti, P. A. O.; Mitliagkas, I.; and Guibas, L. J. 2017. Representation learning and adversarial generation of $3 \mathrm{~d}$ point clouds. CoRR.

Eckstein, I.; Pons, J.-P.; Tong, Y.; Kuo, C.-C.; and Desbrun, M. 2007. Generalized surface flows for mesh processing. In Proceedings of the fifth Eurographics symposium on Geometry processing. Eurographics Association.

Everingham, M.; Van Gool, L.; Williams, C.; Winn, J.; and Zisserman, A. 2011. The pascal visual object classes challenge 2012 (voc2012) results (2012). In URL http://www. pascal-network. org/challenges/VOC/voc2011/workshop/index. html.

Fan, H.; Su, H.; and Guibas, L. 2017. A point set generation network for $3 \mathrm{~d}$ object reconstruction from a single image. IEEE International Conference on Computer Vision (ICCV).

Girdhar, R.; Fouhey, D. F.; Rodriguez, M.; and Gupta, A. 2016. Learning a predictable and generative vector representation for objects. In $E C C V$.

Ioffe, S., and Szegedy, C. 2015. Batch normalization: Accelerating deep network training by reducing internal covariate shift. In International Conference on Machine Learning (ICML).

Kalogerakis, E.; Averkiou, M.; Maji, S.; and Chaudhuri, S. 2017. 3d shape segmentation with projective convolutional networks. CVPR 2017.

Kingma, D., and Ba, J. 2014. Adam: A method for stochastic optimization. arXiv preprint arXiv:1412.6980.

Lin, C.-H.; Kong, C.; and Lucey, S. 2018. Learning efficient point cloud generation for dense $3 \mathrm{~d}$ object reconstruction. In AAAI Conference on Artificial Intelligence (AAAI).

Liu, J.; Wang, J.; Fang, T.; Tai, C.-L.; and Quan, L. 2015. Higher-order crf structural segmentation of $3 \mathrm{~d}$ reconstructed surfaces. In IEEE International Conference on Computer Vision.

Park, E.; Yang, J.; Yumer, E.; Ceylan, D.; and Berg, A. C. 2017. Transformation-grounded image generation network for novel 3d view synthesis. In IEEE Conference on Computer Vision and Pattern Recognition (CVPR), 702-711. IEEE.
Pons, J.-P.; Keriven, R.; and Faugeras, O. 2007. Multi-view stereo reconstruction and scene flow estimation with a global image-based matching score. International Journal of Computer Vision (IJCV) 72(2):179-193.

Pontes, J. K.; Kong, C.; Sridharan, S.; Lucey, S.; Eriksson, A.; and Fookes, C. 2017. Image2mesh: A learning framework for single image $3 \mathrm{~d}$ reconstruction. arXiv preprint arXiv:1711.10669.

Qi, C. R.; Su, H.; Mo, K.; and Guibas, L. J. 2017. Pointnet: Deep learning on point sets for $3 \mathrm{~d}$ classification and segmentation. IEEE Conference on Computer Vision and Pattern Recognition (CVPR).

Shin, D.; Fowlkes, C. C.; and Hoiem, D. 2018. Pixels, voxels, and views: A study of shape representations for single view $3 \mathrm{~d}$ object shape prediction. arXiv preprint arXiv:1804.06032.

Soltani, A. A.; Huang, H.; Wu, J.; Kulkarni, T. D.; and Tenenbaum, J. B. 2017. Synthesizing 3d shapes via modeling multi-view depth maps and silhouettes with deep generative networks. In CVPR 2017.

Tatarchenko, M.; Dosovitskiy, A.; and Brox, T. 2016. Multiview $3 \mathrm{~d}$ models from single images with a convolutional network. In European Conference on Computer Vision (ECCV).

Tulsiani, S.; Zhou, T.; Efros, A. A.; and Malik, J. 2017. Multi-view supervision for single-view reconstruction via differentiable ray consistency. IEEE Conference on Computer Vision and Pattern Recognition (CVPR).

Wang, J.; Fang, T.; Su, Q.; Zhu, S.; Liu, J.; Cai, S.; Tai, C.L.; and Quan, L. Image-based building regularization using structural linear features. IEEE Transactions on Visualization Computer Graphics.

Wu, J.; Zhang, C.; Xue, T.; Freeman, B.; and Tenenbaum, J. 2016. Learning a probabilistic latent space of object shapes via 3d generative-adversarial modeling. In Advances in Neural Information Processing Systems (NIPS).

Wu, J.; Wang, Y.; Xue, T.; Sun, X.; Freeman, B.; and Tenenbaum, J. 2017. Marrnet: 3d shape reconstruction via 2.5 d sketches. In Advances in Neural Information Processing Systems (NIPS).

Yan, X.; Yang, J.; Yumer, E.; Guo, Y.; and Lee, H. 2016. Perspective transformer nets: Learning single-view 3d object reconstruction without $3 \mathrm{~d}$ supervision. In Advances in Neural Information Processing Systems(NIPS).

Zhou, T.; Tulsiani, S.; Sun, W.; Malik, J.; and Efros, A. A. 2016. View synthesis by appearance flow. In ECCV.

Zhu, R.; Galoogahi, H. K.; Wang, C.; and Lucey, S. 2017. Rethinking reprojection: Closing the loop for pose-aware shape reconstruction from a single image. In IEEE International Conference on Computer Vision (ICCV). IEEE. 